\title{
Increased Incidence of Antimicrobial-Resistant Nontyphoidal Salmonella Infections, United States, 2004-2016
}

\author{
Felicita Medalla, Weidong Gu, Cindy R. Friedman, Michael Judd, \\ Jason Folster, Patricia M. Griffin, Robert M. Hoekstra
}

\begin{abstract}
Salmonella is a major cause of foodborne illness in the United States, and antimicrobial-resistant strains pose a serious threat to public health. We used Bayesian hierarchical models of culture-confirmed infections during 2004-2016 from 2 Centers for Disease Control and Prevention surveillance systems to estimate changes in the national incidence of resistant nontyphoidal Salmonella infections. Extrapolating to the United States population and accounting for unreported infections, we estimated a $40 \%$ increase in the annual incidence of infections with clinically important resistance (resistance to ampicillin or ceftriaxone or nonsusceptibility to ciprofloxacin) during 2015-2016 ( $\approx 222,000$ infections) compared with 20042008 ( $\approx 159,000$ infections). Changes in the incidence of resistance varied by serotype. Serotypes I 4,[5],12:i:- and Enteritidis were responsible for two thirds of the increased incidence of clinically important resistance during 20152016. Ciprofloxacin-nonsusceptible infections accounted for more than half of the increase. These estimates can help in setting targets and priorities for prevention.
\end{abstract}

$\mathrm{N}$ Tontyphoidal Salmonella infections cause an estimated 1.2 million illnesses, 23,000 hospitalizations, and 450 deaths each year in the United States (1). Although most infections result in self-limited illness, antimicrobial treatment is recommended for patients with severe infection or at high risk for complications (2). Antimicrobial-resistant Salmonella infections can cause adverse clinical outcomes, including increased rates of hospitalization, bloodstream infection, other invasive illnesses, and death (3-7).

Nontyphoidal Salmonella infections can be acquired during international travel, from contaminated

Author affiliation: Centers for Disease Control and Prevention, Atlanta, Georgia, USA

DOI: https://doi.org/10.3201/eid2706.204486 food and water, through animal contact, and from environmental sources (e.g., wetlands and irrigation water) (8-13). Antimicrobial-resistant infections have been linked to various food and animal sources $(3,14,15)$. In 2015 and previous years, 5 commonly isolated serotypes (Enteritidis, Typhimurium, Newport, I 4,[5],12:i:- and Heidelberg) accounted for more than half of antimicrobial-resistant Salmonella infections in the United States (16-20). The distribution of antimicrobial-resistant infections caused by some of these common serotypes varied by region $(21,22)$.

In a previous study, we found that an estimated annual average of 6,200 culture-confirmed infections were resistant to ceftriaxone or ampicillin or nonsusceptible to ciprofloxacin during 2004-2014 (20). For the study described in this article, we used the same modeling approach and data sources to estimate changes in incidence. We estimated the contribution of the 5 major serotypes to changes in incidence and describe differences by geographic region. We extrapolated findings to the United States population to provide estimates to help set targets and priorities for reducing antimicrobial resistance among nontyphoidal Salmonella.

\section{Methods}

\section{Laboratory-Based Enteric Disease Surveillance}

Public health laboratories in 50 states and many local health departments receive human Salmonella isolates from clinical laboratories and report serotype information to the Centers for Disease Control and Prevention (CDC) through Laboratory-Based Enteric Disease Surveillance (LEDS) (17). We excluded serotypes Typhi, Paratyphi A, Paratyphi B (tartratenegative), and Paratyphi C, which account for $<1 \%$ of 
Salmonella infections in the United States, and whose only known reservoir are humans $(2,16,17,23)$. In this article, we use the term Salmonella to refer to nontyphoidal Salmonella.

\section{National Antimicrobial Resistance Monitoring System}

The National Antimicrobial Resistance Monitoring System (NARMS) is a collaboration among CDC, the US Food and Drug Administration, the US Department of Agriculture, and state and local health departments to monitor resistance among enteric bacteria isolated from humans, retail meat, and food animals $(16,24)$. Public health laboratories in 50 state and 4 local health departments submit every 20th Salmonella isolate received from clinical laboratories to CDC for antimicrobial drug susceptibility testing $(16,19,24)$.

During 2004-2016, CDC tested Salmonella isolates for susceptibility to agents representing 8-9 antimicrobial classes: aminoglycosides, $\beta$-lactam/ $\beta$-lactamase inhibitors, cephems, macrolides (tested since 2011), penicillins, quinolones, folate pathway inhibitors, phenicols, and tetracyclines (16). MICs were determined by broth microdilution with Sensititer (ThermoFisher, https://www.thermofisher. com) and interpreted using criteria from the Clinical and Laboratory Standards Institute (CLSI) when available $(7,16)$. Using CLSI criteria, we defined ceftriaxone resistance as $\mathrm{MIC} \geq 4 \mu \mathrm{g} / \mathrm{mL}$, ampicillin resistance as MIC $\geq 32 \mu \mathrm{g} / \mathrm{mL}$, and nonsusceptibility to ciprofloxacin as MIC $\geq 0.12 \mu \mathrm{g} / \mathrm{mL}$. The ciprofloxacin definition includes both resistant and intermediate CLSI categories because Salmonella infections with intermediate susceptibility to ciprofloxacin have been associated with poor treatment outcomes $(6,7,16)$.

\section{Resistance Categories}

We defined clinically important resistance as resistance to ceftriaxone, nonsusceptibility to ciprofloxacin, or resistance to ampicillin on the basis of the following criteria: third-generation cephalosporins (e.g., ceftriaxone) and fluoroquinolones (e.g., ciprofloxacin) are used for empiric treatment of severe infections; fluoroquinolones are not recommended for children; and ampicillin is useful for susceptible infections (2). We defined and ranked from highest to lowest 3 mutually exclusive categories of clinically important resistance (Appendix Figure 1, https:// wwwnc.cdc.gov/EID/article/27/6/20-4486-App1. pdf) (20): ceftriaxone/ampicillin resistance (because all ceftriaxone-resistant isolates are ampicillin-resistant); ciprofloxacin nonsusceptibility (nonsusceptible to ciprofloxacin but susceptible to ceftriaxone); and ampicillin-only resistance (ampicillin-resistant but susceptible to ceftriaxone and ciprofloxacin). We included ciprofloxacin-nonsusceptible isolates that were ceftriaxone-resistant in the ceftriaxone/ampicillin category because they are of greatest public health and treatment concern. Many isolates in each category had resistance to other agents. We defined multidrug resistance as resistance to $\geq 3$ classes of antimicrobial agents $(16,19)$.

\section{Bayesian Hierarchical Model to Estimate Changes}

We used 2004-2016 data from LEDS, NARMS, and the US Census Bureau as input in the models $(16,17,25)$. For LEDS, we used the number of culture-confirmed infections by state and year (state-year). We combined serotyped isolates other than Enteritidis, Typhimurium, Newport, I 4,[5],12:i:-, and Heidelberg into an "other" category. We assigned unserotyped and partially serotyped isolates from each state into the 6 serotype categories (Enteritidis, Typhimurium, Newport, I 4,[5],12:i:-, Heidelberg, and other) on the basis of the average proportion of serotyped isolates in each category from 2004-2016. For NARMS, we used resistance proportions among fully serotyped isolates per state-year. We used US Census population data for each state-year to express incidence per 100,000 persons per year (25).

A similar Bayesian hierarchical model approach was used from a previous study to estimate the incidence of resistant infections $(20,26)$. However, we found a Poisson model for LEDS data better captured the uncertainty of Salmonella incidence observed at the state-year level instead of the normal distribution used in our previous study (20). The model incorporated the random effects of state, year, and state-year interaction to borrow strength from contiguous states and previous years $(20,26-$ 28). Alaska and Hawaii were excluded because they are not adjacent to any state; the District of Columbia was also excluded, which began submitting isolates to NARMS in $2008(16,19,20)$. We used an approach similar to our previous study to make adjustments for data from Florida, which reported low numbers of isolates compared with its 6 closest states $(17,18,20)$.

We applied the models to generate estimates (referred to as posterior estimates) for Salmonella infection incidence rates, resistance proportions, and resistant infection incidence rates (referred to as resistance incidence) by state-year for each of the 6 serotype categories by using Markov chain Monte Carlo simulations $(20,26-28)$. For each serotype category, we estimated resistance incidence for overall 
clinically important resistance, the 3 mutually exclusive categories of clinically important resistance, and multidrug resistance. For all Salmonella, we calculated overall estimates by summing estimates across the 6 serotype categories. We calculated state-year resistance incidence estimates per 100,000 persons per year as estimated incidence for state-year $\times$ estimated resistance proportion for state-year. For estimation of resistance incidence by geographic region, we used the 4 US Census region categories (Midwest, Northeast, South, and West) and aggregated posterior estimates of resistance incidence by year for all states in each region (25). For each resistance category, we calculated mean estimates and 95\% credible intervals (CrIs) from posterior estimates and mean crude rates by year for the 48 states and those stratified by serotype and region categories
(Figure 1; Appendix Figures 2-5) for an overall sideby-side comparison $(20,25,26)$.

To assess changes in resistance incidence, we compared the mean resistance incidence from 2015-2016 with that from two 5-year reference periods during 2004-2016: 2004-2008 and 2010-2014. These reference periods are consistent with those used in NARMS annual reports to assess changes in resistance percentages (16). All 50 states have participated in NARMS since 2003; the 2004-2008 period is the early years of nationwide participation and the 2010-2014 period is the recent past. For each resistance and serotype category, we calculated the difference between the posterior estimates of resistance incidence for 20152016 and those for each year in the 5-year reference periods for each region to obtain the mean difference and the 95\% CrIs. We did not assume homogeneous
- Estimated resistance incidence

- Crude resistance incidence

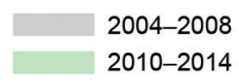

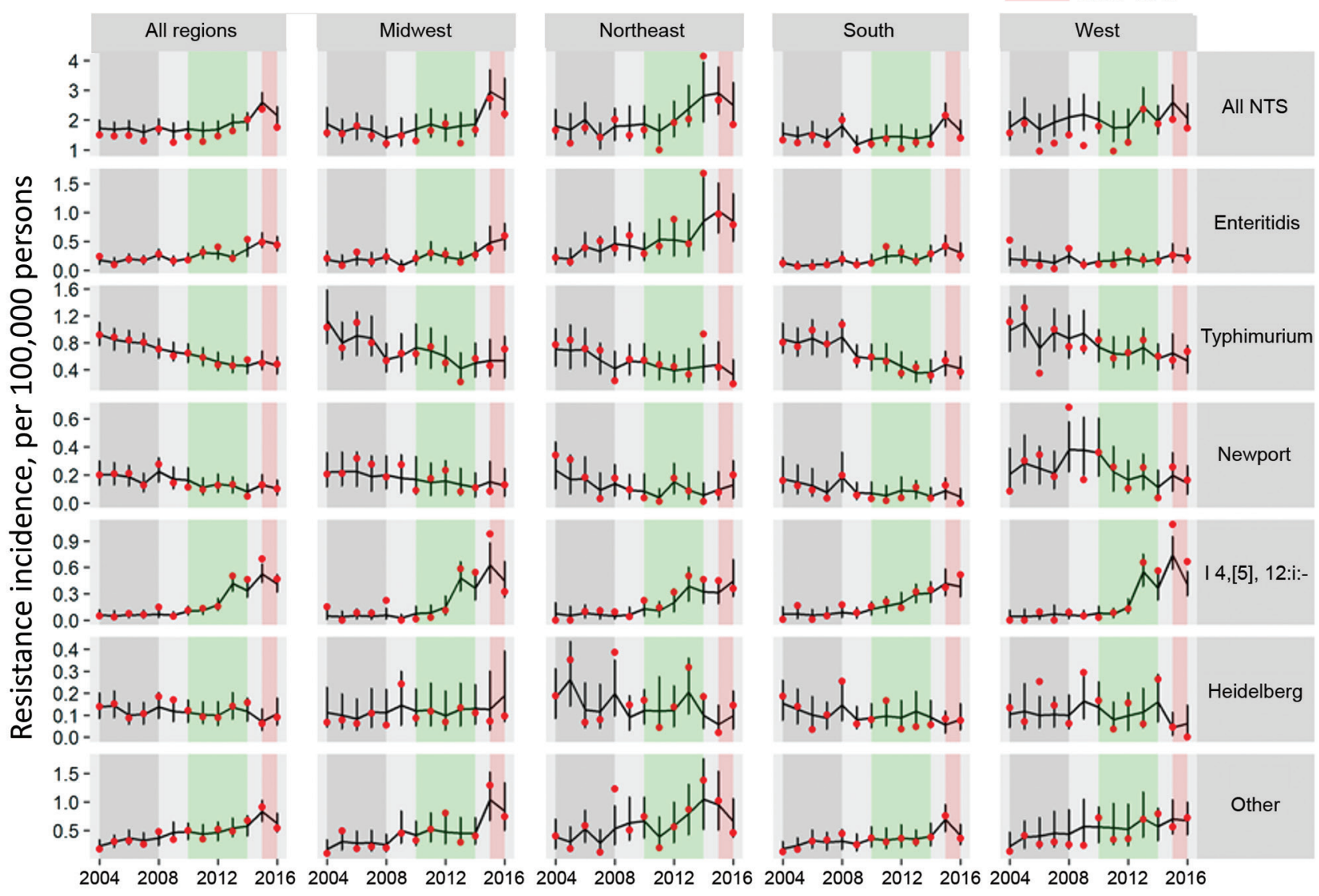

Figure 1. Estimated annual incidence of culture-confirmed nontyphoidal Salmonella infections with any clinically important resistance, by serotype and region, United States, 2004-2016. Estimated changes in resistance incidence (mean and 95\% credible intervals of the posterior differences per 100,000 persons per year) were derived using Bayesian hierarchical models. Crude resistance incidence rates were derived by multiplying infection incidence and resistance proportion for state-year. Any clinically important resistance was defined as resistant to ceftriaxone, resistant to ampicillin, or ciprofloxacin nonsusceptible. The "other" category comprised serotypes other than Enteritidis, Typhimurium, Newport, I 4,[5],12:i:-, and Heidelberg. US Census regions were used to define 4 geographic regions. NTS, all nontyphoidal Salmonella serotypes. 
rates across multiple years using this approach. For all Salmonella, we calculated the change in resistance incidence, which represents the net change (increase or decrease), for each resistance category, by summing the estimated changes derived for the 6 serotype categories. We describe statistically significant changes (i.e., in which the $95 \%$ CrIs do not include 0).

\section{Extrapolating to the US Population}

We multiplied the mean estimates of culture-confirmed infections by 29 , which is the estimated number of total infections for every culture-confirmed infection in the general population, to estimate the total number of resistant infections for each period and changes in total resistant per 100,000 persons per year during 2015-2016 compared with the reference periods for each resistance category (1). We used the average 2015-2016 population estimates for the 50 states (322 million) to extrapolate to the US population (25).

\section{Results}

During 2004-2016, public health laboratories of state and participating local health departments in the 48 contiguous states reported 539,862 culture-confirmed Salmonella infections to LEDS (Appendix Table 1). Among the isolates from these infections, $89 \%$ were serotyped; the most common were Enteritidis (20\%), Typhimurium (16\%), Newport (11\%), I 4,[5],12:i:- (4\%), and Heidelberg (4\%). Public health laboratories in the 48 states submitted 28,265 isolates to NARMS. Of these isolates, $98 \%$ were serotyped; the most common were Enteritidis (19\%), Typhimurium (16\%), Newport (11\%), I 4,[5],12:i:- (4\%), and Heidelberg (4\%).
Clinically Important Resistance and Multidrug Resistance During 2004-2016, clinically important resistance was detected in $3,546(12.5 \%)$ of 28,265 isolates (Table 1; Appendix Figure 1). Ampicillin-only resistance was detected in 1,857 (6.6\%) isolates, ciprofloxacin nonsusceptibility in $854(3.0 \%)$, and ceftriaxone/ampicillin resistance in $835(3.0 \%)$. Only $78(0.3 \%)$ isolates were resistant to ceftriaxone and nonsusceptible to ciprofloxacin; these isolates were included in the 835 categorized as ceftriaxone/ampicillin-resistant. Most (>90\%) ciprofloxacin-nonsusceptible isolates had MICs within the intermediate range, 0.12-0.5 (Table 1; Appendix Figure 6).

Of the 28,265 isolates, 2,912 (10.3\%) were multidrug resistant (MDR). Of these, 2,633 (90\%) had clinically important resistance, which accounted for $74 \%$ of the 3,546 isolates with clinically important resistance.

\section{Incidence by Year and Region, 2004-2016}

For each resistance category, the trend lines were smoother with model-derived annual estimates of resistance incidence compared with crude rates, particularly when stratified by serotype and region (Figure 1; Appendix Figures 2-5). Crude rates tended to be lower than model-derived estimates because many state-year resistance proportions used in calculating crude rates were 0 because of small sample sizes, whereas the model tended to pull estimates away from 0 . Overall, most crude rates were within modelderived 95\% CrIs.

\section{Resistance Incidence, 2015-2016}

During 2015-2016, the mean annual incidence was 2.38 (95\% CrI 1.93-2.86)/100,000 persons for clinically important resistant infections and 1.83 (95\%

\footnotetext{
Table 1. Number and percentage of antimicrobial-resistant nontyphoidal Salmonella isolates, by serotype and resistance category, United States, 2004-2016*

\begin{tabular}{|c|c|c|c|c|c|c|c|c|}
\hline \multirow[b]{2}{*}{ Resistance category } & \multicolumn{8}{|c|}{ No. $(\%)$ isolates } \\
\hline & $\begin{array}{c}\text { Enteritidis, } \\
\mathrm{n}=5,206\end{array}$ & $\begin{array}{c}\text { Typhimurium, } \\
n=4,404\end{array}$ & $\begin{array}{l}\text { Newport, } \\
\mathrm{n}=3,140\end{array}$ & $\begin{array}{c}\text { 4,[5],12:i:-, } \\
n=1,158\end{array}$ & $\begin{array}{c}\text { Heidelberg, } \\
\mathrm{n}=974\end{array}$ & $\begin{array}{l}\text { Other fully } \\
\text { serotyped, } \\
\mathrm{n}=12,878\end{array}$ & $\begin{array}{l}\text { Not fully } \\
\text { serotyped, } \\
n=505\end{array}$ & $\begin{array}{c}\text { Total } \\
\text { nontyphoidal } \\
\text { Salmonella, } \\
\mathrm{N}=28,265\end{array}$ \\
\hline $\begin{array}{l}\text { Any clinically important } \\
\text { resistance } \dagger\end{array}$ & $548(10.5)$ & $1,197(27.2)$ & $284(9.0)$ & $389(33.6)$ & $240(24.6)$ & $843(6.5)$ & $45(8.9)$ & $3,546(12.5)$ \\
\hline Multidrug resistance $\ddagger$ & $114(2.2)$ & $1,178(26.7)$ & $271(8.6)$ & $382(33.0)$ & $204(20.9)$ & $727(5.6)$ & $36(7.1)$ & $2,912(10.3)$ \\
\hline Amp-only§ & $152(2.9)$ & $897(20.4)$ & $30(1.0)$ & $319(27.5)$ & $120(12.3)$ & $311(2.4)$ & $28(5.5)$ & $1,857(6.6)$ \\
\hline Cef/Amp§ণ & $15(0.3)$ & $212(4.8)$ & $237(7.5)$ & $39(3.4)$ & 116 (11.9) & $212(1.6)$ & .8) & $835(3.0)$ \\
\hline Ciproß\# & $381(7.3)$ & $88(2.0)$ & $17(0.5)^{\prime}$ & $31(2.7)$ & $4(0.4)$ & $320(2.5)$ & $13(2.6)$ & $854(3.0)$ \\
\hline \multicolumn{9}{|c|}{$\begin{array}{l}\text { *Amp-only, resistant to ampicillin (MIC } \geq 32 \mu \mathrm{g} / \mathrm{mL} \text { ) but susceptible to ceftriaxone and ciprofloxacin; Cef/Amp, resistant to ceftriaxone }(\mathrm{MIC} \geq 4 \mu \mathrm{g} / \mathrm{mL}) \text { and } \\
\text { ampicillin; Cipro, nonsusceptible to ciprofloxacin (MIC } \geq 0.12 \mu \mathrm{g} / \mathrm{mL} \text { ) but susceptible to ceftriaxone; NTS, nontyphoidal Salmonella, which includes isolates } \\
\text { serotyped as Enteritidis, Typhimurium, Newport, I 4,[5],12:i:-, and Heidelberg, isolates serotyped as other than those } 5 \text {, and those not fully serotyped. } \\
\text { †Includes any of the } 3 \text { clinically important resistance patterns (i.e., resistant to ceftriaxone, resistant to ampicillin, or nonsusceptible to ciprofloxacin). } \\
\text { Isolates might have resistance to other agents tested. } \\
\text { †Resistant to } \geq 3 \text { classes of antimicrobial agents. } \\
\text { \$Amp-only, Ceff/Amp, and Cipro are mutually exclusive categories of clinically important resistance. } \\
\text { TOf the } 835 \text { isolates with Cef/Amp resistance, } 78 \text { (0.3\% of all nontyphoidal Salmonella isolates) were nonsusceptible to ciprofloxacin. Of the } 78 \text { isolates, } \\
71 \text { (91\%) had ciprofloxacin MICs within the intermediate range (i.e., } 0.12-0.5) \text { (Appendix Figure } 6 \text {, https://wwwnc.cdc.gov/EID/article/27/6/20-4486- } \\
\text { App1.pdf). These } 78 \text { isolates were not included in the Cipro category. } \\
\text { \#Of the } 854 \text { isolates, } 785 \text { (92\%) had ciprofloxacin MICs within the intermediate range (Appendix Figure } 6 \text { ). }\end{array}$} \\
\hline
\end{tabular}
}


CrI 1.45-2.25)/100,000 persons for MDR infections (Table 2). The 5 major serotypes accounted for $69 \%$ of infections with clinically important resistance and $66 \%$ with multidrug resistance.

\section{Changes in Resistance Incidence, 2015-2016 versus Reference Periods}

The mean annual incidence of infections with any clinically important resistance increased during 2015-2016 compared with 2004-2008; there was no significant change compared with 2010-2014 (Table 2; Figures 2 and 3 ). Among the resistance categories, the mean annual incidence of ciprofloxacin-nonsusceptible Salmonella infections increased during 2015-2016 compared with both reference periods.

\section{Changes in Resistance Incidence, 2015-2016 versus 2004-2008}

The mean annual incidence of Salmonella infections with clinically important resistance increased by 0.68 (95\% CrI 0.13-1.24)/100,000 persons (Table $2)$. By census region, a significant increase in resistance only occurred in the Midwest (Figure 2). By serotype, I 4,[5],12:i:- had an incidence increase of $0.41(95 \%$ CrI $0.27-0.56) / 100,000$ persons, accounting for $37 \%$ of the increase in clinically important resistant Salmonella infections (Appendix Table 2). The incidence of resistant I 4,[5],12:i:- infections increased significantly in all 4 regions, with highest increase in the West and Midwest. Enteritidis infections with clinically important resistance increased by $0.29 \quad(95 \% \quad \mathrm{CrI} \quad 0.12-$ $0.47) / 100,000$ persons, accounting for $26 \%$ of the increase in resistant infections. This increase was significant in 3 regions, with highest increase in the Northeast. Infections with clinically important resistance caused by serotypes categorized as other increased by 0.41 (95\% CrI 0.12-0.72)/100,000 persons, accounting for $37 \%$ of the increase in resistant infections (Figure 2; Appendix Table 2). Typhimurium infections with clinically important resistance decreased ( -0.33 [ $95 \% \mathrm{CrI}-0.58$ to -0.07$]$ / 100,000 persons).

Although no significant changes were noted in the mean annual incidence of Salmonella infections with multidrug or ampicillin-only resistance, some serotypes did change (Figure 2; Appendix Table 2). MDR I 4,[5],12:i:- infections increased $(0.40$ [95\% CrI $0.24-$ $0.56] / 100,000$ persons); this change was significant in all 4 regions, with highest increase in the West and Midwest. The incidence of MDR Enteritidis infections also increased (0.13 [95\% CrI 0.04-0.23]/100,000 persons). We observed a decrease in Typhimurium infections with multidrug resistance $(-0.37$ [95\% CrI -0.59 to -0.14$] / 100,000$ persons) and ampicillin-only resistance $(-0.35$ [95\% CrI -0.61 to -0.10$] / 100,000$ persons). Serotype I 4,[5],12:i:- infections with ampicillin-only resistance increased $(0.35$ [95\% CrI 0.21-0.50]/100,000 persons); this change was significant in all 4 regions, with highest increase in the West and Midwest.

The mean annual incidence of ciprofloxacinnonsusceptible Salmonella infections increased by 0.41

Table 2. Estimated incidence and changes in the incidence of antimicrobial-resistant culture-confirmed nontyphoidal Salmonella infections, by resistance category, United States, 2015-2016 versus 2004-2008 and 2010-2014* Mean $(95 \% \mathrm{Crl})$

\begin{tabular}{|c|c|c|c|c|c|}
\hline \multirow[b]{4}{*}{ Resistance category } & \\
\hline & \multirow{2}{*}{\multicolumn{3}{|c|}{ Resistance incidence, per 100,000 persons per year† }} & \multicolumn{2}{|c|}{$\begin{array}{l}\text { Change in resistance incidence, per } \\
100,000 \text { persons per year }\end{array}$} \\
\hline & & & & \multirow{2}{*}{$\begin{array}{l}2015-2016 \\
\text { vs. } 2004-2008\end{array}$} & \multirow{2}{*}{$\begin{array}{l}2015-2016 \\
\text { vs. } 2010-2014\end{array}$} \\
\hline & $2015-2016$ & 2004-2008 & $2010-2014$ & & \\
\hline $\begin{array}{l}\text { Any clinically important } \\
\text { resistance } \S\end{array}$ & $2.38(1.93-2.86)$ & $1.70(1.44-1.98)$ & $1.78(1.46-2.15)$ & $0.68(0.13$ to 1.24$) \ddagger$ & $0.60(-0.002$ to 1.20$)$ \\
\hline Multidrug resistanceף & $1.83(1.45-2.25)$ & $1.51(1.27-1.79)$ & $1.42(1.16-1.70)$ & $0.32(-0.17$ to 0.82$)$ & $0.41(-0.07$ to 0.92$)$ \\
\hline Amp-only§ & $1.19(0.85-1.56)$ & $1.00(0.78-1.25)$ & $0.96(0.73-1.21)$ & $0.19(-0.25$ to 0.63$)$ & $0.23(-0.21$ to 0.67$)$ \\
\hline Cef/Amp§ & $0.49(0.37-0.65)$ & $0.43(0.31-0.58)$ & $0.42(0.30-0.56)$ & $0.06(-0.13$ to 0.26$)$ & $0.08(-0.11$ to 0.26$)$ \\
\hline Cipro§ & $0.70(0.55-0.88)$ & $0.29(0.19-0.41)$ & $0.41(0.26-0.64)$ & $0.41(0.22$ to 0.61$) \ddagger$ & $0.29(0.02-0.52) \ddagger$ \\
\hline \multicolumn{6}{|c|}{$\begin{array}{l}\text { *Amp-only, resistant to ampicillin (MIC } \geq 32 \mu \mathrm{g} / \mathrm{mL} \text { ) but susceptible to ceftriaxone and ciprofloxacin; BHM, Bayesian hierarchical model; Cef/Amp, resis } \\
\text { to ceftriaxone (MIC } \geq 4 \mu \mathrm{g} / \mathrm{mL} \text { ) and ampicillin; Cipro, nonsusceptible to ciprofloxacin (MIC } \geq 0.12 \mu \mathrm{g} / \mathrm{mL} \text { ) but susceptible to ceftriaxone; Crl, credible } \\
\text { interval. } \\
\text { †Mean estimates of resistance incidence and } 95 \% \text { Crls were derived using BHMs. Serotypes other than Enteritidis, Typhimurium, Newport, I } 4 \text {,[5],12: } \\
\text { and Heidelberg were combined into the "other" category. For all nontyphoidal Salmonella, estimates were derived by summing those for the } 6 \text { serotyp } \\
\text { categories. State-year data were too sparse to use in the BHMs to estimate mean resistance incidence for Cef/Amp among Enteritidis and Cipro amo } \\
\text { Newport and Heidelberg ( } 4,5 \text {, and } 6 \text { Enteritidis isolates, } 7,2 \text {, and } 8 \text { Newport isolates, and } 0,1 \text {, and } 3 \text { Heidelberg isolates in } 2015-2016,2004-2008 \text {, } \\
2010-2014 \text {, respectively). } \\
\text { †Resistance incidence in } 2015-2016 \text { was compared with that from } 2 \text { reference periods, } 2004-2008 \text { and } 2010-2015 \text { (e.g., increase if } 2015-2016> \\
\text { reference). Mean changes are reported as significant (bold font) if the } 95 \% \text { Crls (rounded to } 2 \text { decimals) do not include } 0 \text {. } \\
\text { §An overall category of clinically important resistance includes any of } 3 \text { resistance patterns (i.e., resistant to ceftriaxone, resistant to ampicillin, or } \\
\text { nonsusceptible to ciprofloxacin). Amp-only, Cef/Amp, and Cipro are mutually exclusive categories of clinically important resistance. Isolates with any } \\
\text { clinically important resistance might have resistance to other agents tested. Model estimates for overall clinically important resistance were derived } \\
\text { separately and might differ from the sum of estimates for the } 3 \text { mutually exclusive categories. }\end{array}$} \\
\hline
\end{tabular}



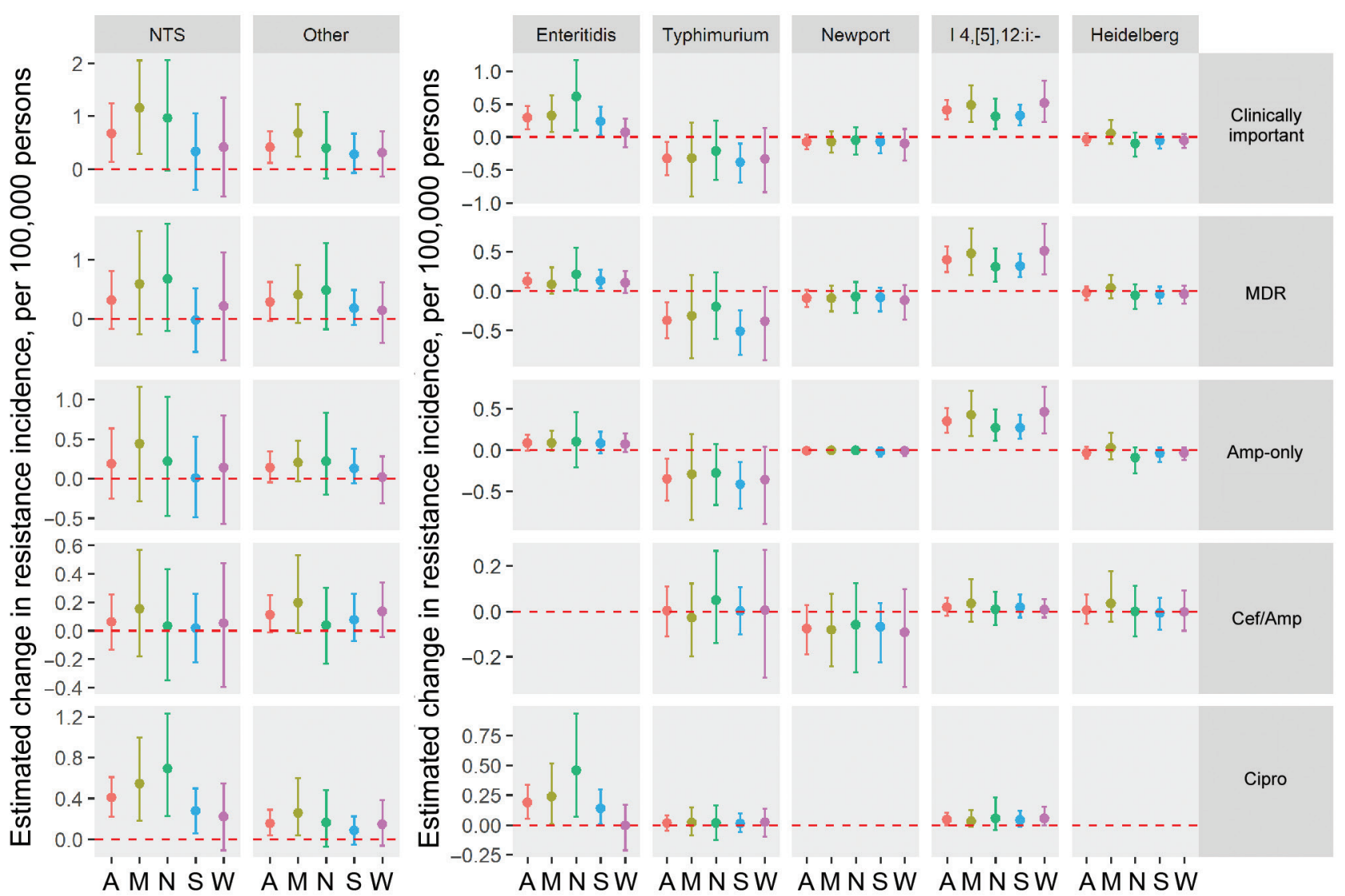

Figure 2. Estimated changes in the incidence of resistant culture-confirmed nontyphoidal Salmonella infections, by serotype, resistance category, and geographic region, United States, 2015-2016 versus 2004-2008. Estimated changes in resistance incidence (mean and $95 \%$ credible intervals of the posterior differences per 100,000 persons/year) were derived using Bayesian hierarchical models. Amp-only, Cef/Amp, and Cipro are mutually exclusive categories of clinically important resistance: Amp-only, resistant to ampicillin but susceptible to ceftriaxone and ciprofloxacin; Cef/Amp, resistant to ceftriaxone and ampicillin; Cipro, nonsusceptible to ciprofloxacin but susceptible to ceftriaxone. Isolates in each category might have resistance to other agents. Multidrug resistance was defined as resistance to $\geq 3$ classes of antimicrobial agents. The "other" category comprised serotypes other than Enteritidis, Typhimurium, Newport, I 4,[5],12:i:-, and Heidelberg. US Census regions were used to define 4 geographic regions (A, all regions; M, Midwest; N, Northeast; S, South; W, West). MDR, multidrug resistant. NTS, all nontyphoidal Salmonella serotypes.

(95\% CrI 0.22-0.61)/100,000 persons (Table 2). Ciprofloxacin-nonsusceptible Enteritidis infections increased by 0.19 (95\% CrI 0.05-0.34)/100,000 persons, accounting for $47 \%$ of the increase in these infections (Appendix Table 2). This increase was significant in 3 regions, most notably in the Northeast (Figure 2). Ciprofloxacin-nonsusceptible infections caused by serotypes categorized as other increased by $0.16(95 \%$ CrI $0.04-0.29) / 100,000$ persons, accounting for $38 \%$ of the increase in ciprofloxacin-nonsusceptible infections (Figure 2; Appendix Table 2).

\section{Changes in Resistance Incidence, 2015-2016 versus 2010-2014}

The mean annual incidence of Salmonella infections with clinically important resistance did not change compared with the previous 5 years. However, the mean annual incidence of ciprofloxacin-nonsusceptible Salmonella infections increased by 0.29 (95\% CrI 0.02-0.52)/100,000 persons (Table 2); by region, the increase was significant only in the Midwest (Figure 3). Ciprofloxacin-nonsusceptible Enteritidis infections increased by $0.16(95 \% \mathrm{CrI}$ $0.02-0.32) / 100,000$ persons, accounting for $57 \%$ of the increase in ciprofloxacin-nonsusceptible infections (Appendix Table 3).

\section{Extrapolation to the US population}

Compared with the number of infections for 2004-2008, an estimated $\approx 63,000$ more Salmonella infections with clinically important resistance occurred each year during 2015-2016, from an average of $\approx 159,000$ to $\approx 222,000$; more than half were ciprofloxacin-nonsusceptible (Table 3). Compared with the number of infections for 

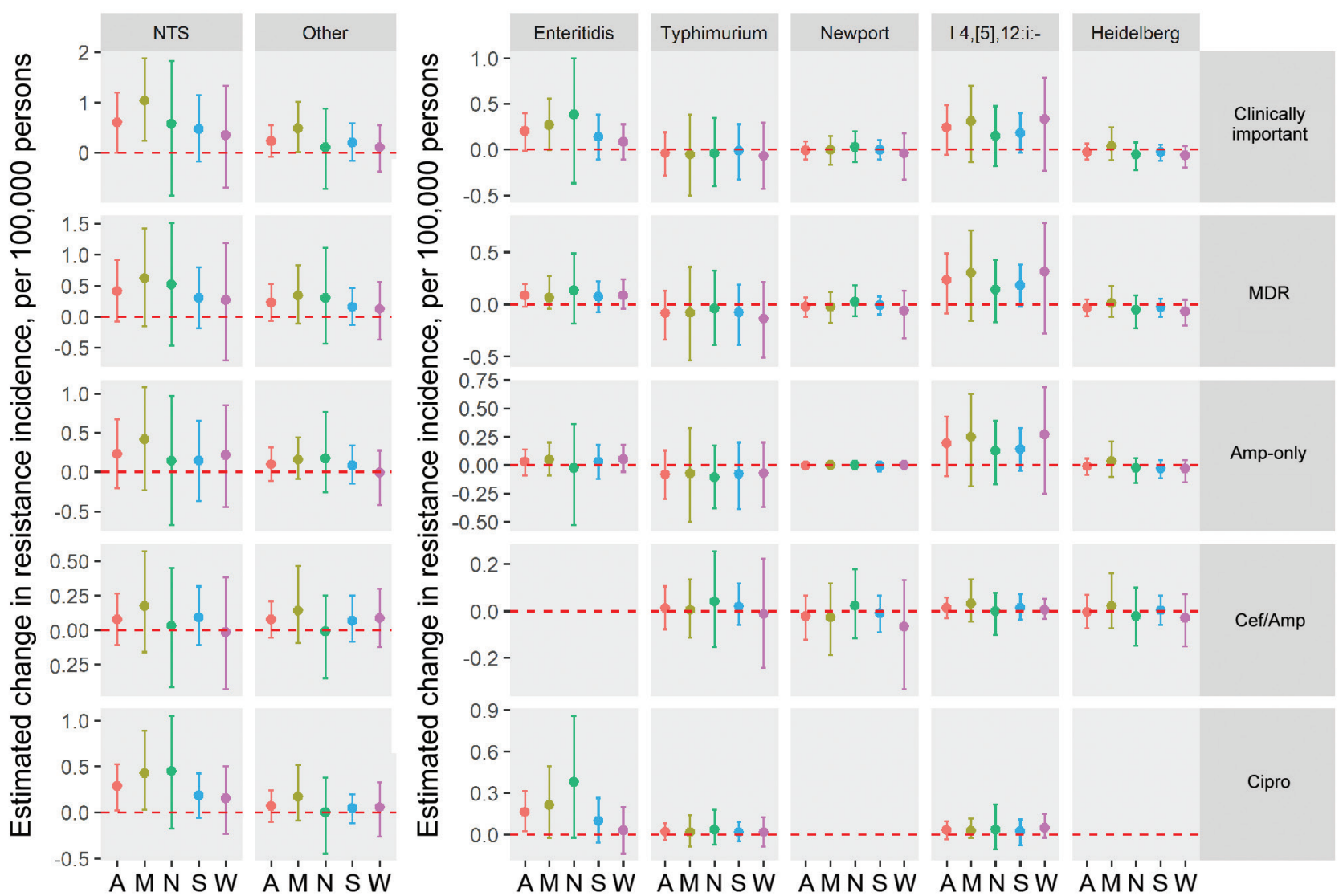

Figure 3. Estimated changes in the incidence of resistant culture-confirmed nontyphoidal Salmonella infections, by serotype, resistance category, and geographic region, United States, 2015-2016 versus 2010-2014. Estimated changes in resistance incidence (mean and $95 \%$ credible intervals of the posterior differences per 100,000 persons/year) were derived using Bayesian hierarchical models. Amp-only, Cef/Amp, and Cipro are mutually exclusive categories of clinically important resistance: Amp-only, resistant to ampicillin but susceptible to ceftriaxone and ciprofloxacin; Cef/Amp, resistant to ceftriaxone and ampicillin; Cipro, nonsusceptible to ciprofloxacin but susceptible to ceftriaxone. Isolates in each category might have resistance to other agents. Multidrug resistance (MDR) was defined as resistance to $\geq 3$ classes of antimicrobial agents. The "other" category comprised serotypes other than Enteritidis, Typhimurium, Newport, I 4,[5], 12:i:-, and Heidelberg. US Census regions were used to define 4 geographic regions (A, all regions; M, Midwest; N, Northeast; S, South; W, West). MDR, multidrug resistant; NTS, all nontyphoidal Salmonella serotypes.

the previous 5 years, an estimated $\approx 56,000$ more Salmonella infections with clinically important resistance occurred each year during 2015-2016; more than half were ciprofloxacin-nonsusceptible.

\section{Discussion}

Our analysis indicates that the incidence of resistant Salmonella infections was higher in 2015-2016 than in earlier periods during 2004-2014. The annual incidence of culture-confirmed infections with clinically important resistance increased by $0.68 / 100,000$ persons, a $40 \%$ increase in the annual number of infections, during 2015-2016 compared with 2004-2008. Serotypes I 4,[5],12:i:- and Enteritidis were responsible for two thirds of this increase. Ciprofloxacinnonsusceptible infections accounted for more than half of the increase. Extrapolating to total infections in the US population using a multiplier to account for unreported infections resulted in an estimated $\approx 63,000$ more infections with clinically important resistance per year during 2015-2016 compared with 2004-2008 (from $\approx 159,000$ to $\approx 222,000$ infections).

The increased incidence of ciprofloxacin-nonsusceptible Salmonella infections during 2015-2016 compared with incidence for both 2004-2008 and 2010-2014 is a concerning trend. Serotype Enteritidis contributed the most to this increase. Although the incidence of infections with Enteritidis, the most common serotype, has not changed significantly in $>10$ years, the percentage of ciprofloxacin-nonsusceptible infections has increased almost steadily $(11,16)$. Chicken and eggs have been the main domestic sources of Enteritidis infections $(29,30)$. About $20 \%$ of Enteritidis infections are linked to international travel, which is an 
important source of ciprofloxacin-nonsusceptible Enteritidis infections $(8,31,32)$.

The incidence of infections with clinically important resistance and ciprofloxacin-nonsusceptibility caused by serotypes categorized as other was higher during 2015-2016 than during 2004-2008. Some of these serotypes are emerging or have concerning levels of resistance, including Dublin, Infantis, Kentucky, Hadar, and Agona $(16,24,33)$. Some have been associated with resistance, invasive illness, or both $(11,19,23,33)$.

The decrease in resistant Typhimurium infections might be related to the simultaneous increase in I 4,[5],12:i- infections, which some call monophasic Typhimurium $(16,18,34)$. In the 1990s, MDR Typhimurium infections increased markedly in Europe and then in the United States $(35,36)$. Most isolates from these infections that underwent phage typing were definitive type $104(14,21,35,36)$. Isolations of this strain have decreased globally; the reasons are not known (36)

Changes in resistance incidence by resistance category and serotype varied by geographic region, with significant increases in most regions for serotypes I 4,[5],12:i:- and Enteritidis. An increase in the incidence of I 4,[5],12:i:- infections with multidrug and ampicillin-only resistance occurred in all 4 regions, with highest increase in the West and Midwest. Pork products have been associated with I 4,[5],12:i:- infections with resistance to ampicillin, sulfonamide, streptomycin, and tetracycline in the West $(34,37)$. The regional pattern of pork consumption has reflected the regional pattern of pork production, which is highest in the Midwest; 8 of the 10 states with the highest production of swine are in the Midwest $(38,39)$. A study showed that MDR I 4,[5],12:i:- strains from swine in the Midwest during 2014-2016 were typically resistant to ampicillin, sulfonamide, streptomycin, and tetracycline and probably part of a European clade that has spread in the United States and elsewhere; these strains harbored plasmid-mediated resistance genes, which can be transmitted horizontally to other bacteria (34). This trend could partly explain the widespread increase in the incidence of MDR I 4,[5],12:i:- infections. International travel could have contributed to an increase in the incidence of ciprofloxacin-nonsusceptible Enteritidis infections, which increased in 3 regions and was highest in the Northeast. International travel has increased since 2014, and residents of northeastern states accounted for more than one third of US travelers during 2015-2016 (40). In the United Kingdom, an increase in these infections has been linked to international travel and imported foods (41). In the United States, ciprofloxacin-nonsusceptible strains of Enteritidis and other serotypes have been isolated from imported seafood (42). Plasmid-mediated quinolone-resistance genes have been detected among ciprofloxacin-nonsusceptible isolates in the United States; these genes might contribute to spread of fluoroquinolone nonsusceptibility (43).

Our use of a Bayesian hierarchical model improved the estimates, as shown by the smoothing of resistance incidence and temporal change lines, by addressing issues related to missing and sparse stateyear data $(20,26)$. Our method of calculating the aver-

\begin{tabular}{|c|c|c|c|c|c|}
\hline \multirow[b]{3}{*}{ Resistance category } & \multirow{2}{*}{\multicolumn{3}{|c|}{ No. infections/yeart }} & \multicolumn{2}{|c|}{ Change in no. infections/year } \\
\hline & & & & \multirow{2}{*}{$\begin{array}{c}2015-2016 \\
\text { vs. } 2004-2008 \\
\end{array}$} & \multirow{2}{*}{$\begin{array}{c}2015-2016 \\
\text { vs. } 2010-2014\end{array}$} \\
\hline & $2015-2016$ & 2004-2008 & 2010-2014 & & \\
\hline Any clinically important resistance & 222,000 & 159,000 & 166,000 & $63,000 \ddagger$ & 56,000 \\
\hline Multidrug resistance & 171,000 & 141,000 & 133,000 & 30,000 & 38,000 \\
\hline Amp-only§ & 111,000 & 93,000 & 90,000 & 18,000 & 21,000 \\
\hline Cef/Amp§ & 46,000 & 40,000 & 39,000 & 6,000 & 7,000 \\
\hline Cipro§ & 65,000 & 27,000 & 38,000 & $38,000 \ddagger$ & $27,000 \ddagger$ \\
\hline
\end{tabular}

${ }^{*} \mathrm{Amp}$-only, resistant to ampicillin (MIC $\geq 32 \mu \mathrm{g} / \mathrm{mL}$ ) but susceptible to ceftriaxone and ciprofloxacin; BHM, Bayesian hierarchical model; Cef/Amp, resistant to ceftriaxone (MIC $\geq 4 \mu \mathrm{g} / \mathrm{mL}$ ) and ampicillin; Cipro, nonsusceptible to ciprofloxacin (MIC $\geq 0.12 \mu \mathrm{g} / \mathrm{mL}$ ) but susceptible to ceftriaxone; Crl, credible interval.

†Point estimates extrapolated to the entire US population were calculated by multiplying mean estimates for culture-confirmed infections (derived using $\mathrm{BHM}$ ) by the multiplier of 29 and the average total U.S. population for $2015-2016$ (322 million). The multiplier of 29 is the mean estimate of the total number of infections for every culture-confirmed nontyphoidal Salmonella infection. The 95\% Crls were not derived. Extrapolated point estimates were rounded to the nearest thousand.

$\ddagger$ Model-derived mean estimates of changes in resistance incidence (Table 2) used to calculate extrapolated estimates are reported as significant if the 95\% Crls do not include 0; the extrapolated estimates corresponding to these BHM-derived estimates are shown in bold font. Although the $95 \%$ Crls of extrapolated estimates were not derived, they can be assumed to include 0 if the $95 \%$ Crls of BHM-derived estimates include 0 .

$\S A n$ overall category of clinically important resistance includes any of 3 resistance patterns (i.e., resistant to ceftriaxone, resistant to ampicillin, or nonsusceptible to ciprofloxacin). Amp-only, Cef/Amp, and Cipro are mutually exclusive categories of clinically important resistance. Isolates with any clinically important resistance might have resistance to other agents tested. Model estimates for overall clinically important resistance were derived separately and might differ from the sum of BHM estimates for the 3 mutually exclusive categories; thus, extrapolated estimates for the overall category might differ from the sum of mutually exclusive categories.

TResistant to $\geq 3$ classes of antimicrobial agents. 
age difference in incidence between groups of years is more refined than approaches using a negative binomial model because it does not assume homogeneous resistance incidence rates across multiple years $(11,44)$. It is therefore less likely to underestimate the variability of estimated changes. However, this analysis is subject to the same limitations described in previous reports, including unmeasured sources of bias and uncertainty derived by combining data from separate unlinked surveillance systems $(20,26)$. Our estimates of significant changes were limited to comparisons with the reference periods used to assess changes in resistance percentages in NARMS annual reports (16). Our choice to compare a recent 2-year period with earlier 5-year periods balanced the need to assess the most current situation with the need for sufficient data to assess significant changes. Because of the low percentage of isolates showing resistance to trimethoprim/sulfamethoxazole $(<3 \%)$ or decreased susceptibility to azithromycin $(<1 \%)$, an important agent used to treat serious infections, we did not provide estimates for these agents $(2,16,18,20)$. We included infections resistant to ceftriaxone and nonsusceptible to ciprofloxacin in the ceftriaxone/ampicillin-resistance category; they represented only $0.3 \%$ of Salmonella isolates submitted to NARMS. The fact that some ciprofloxacin nonsusceptible infections were not included in the ciprofloxacin nonsusceptible category further supports our finding that ciprofloxacin-nonsusceptible infections increased during the study period. Increasing use of culture-independent diagnostic tests by clinical laboratories can change the submission of isolates to public health laboratories and reporting of infections (11); these changes warrant adjustments in future analyses (20).

We multiplied estimates of culture-confirmed infections by 29 to account for undiagnosed infections. However, resistant infections are associated with more severe illness, so they might be more likely to be detected (3-6). Thus, the appropriate multiplier (the ratio of total infections to cultureconfirmed infections) for resistant infections might be $<29$. To calculate undiagnosed Salmonella infections, multipliers of 12 for persons $<5$ years of age and 23 for persons $\geq 65$ years of age have been reported (45). Although children $<5$ years of age have the highest incidence of Salmonella infections, older adults might disproportionately account for resistant infections because they are more likely to have serious illness and be hospitalized (4,5,44-47); therefore, a multiplier of 23 might be an appropriate choice. However, we chose 29 because it was used in a previous estimate of the total number of Salmonella infections in the population (1) and because persons 5-64 years of age account for most cultureconfirmed infections reported to CDC and most isolates with clinically important resistance submitted to NARMS $(4,18,44,45)$. We did not attach uncertainties to the extrapolated total number of resistant infections and changes in that number because uncertainties of the multiplier are not known. Although resistance incidence can vary by demographic subgroup, geographic region, time, and other factors, we did not include additional uncertainties from the extrapolation to the US population using the average 2015-2016 population estimates for the 50 states $(19,21,22,46,47)$.

Estimates of changes in resistance incidence can help identify trends of greatest concern to set priorities for prevention. Analyses that include the varying distributions of infections by demographic subgroups, season, and recent travel could inform serotype-specific, regional, and source-targeted prevention strategies $(5,11,21,22,31,44-48)$. The increasing use of whole-genome sequencing by public health laboratories to characterize Salmonella strains will enhance surveillance of antimicrobialresistant Salmonella from human and nonhuman sources (49). Antimicrobial agents contribute to resistance wherever they are used, including in food animals and humans (50). A One Health approach can help in detecting and controlling antimicrobial resistance, which is a complex and multifaceted problem that affects humans, animals, and the environment (50).

\section{Acknowledgments}

We thank state and local health departments and their public health laboratories for their contributions to the National Antimicrobial Resistance Monitoring System and Laboratory-Based Enteric Disease Surveillance. We acknowledge Sean Browning for his assistance with Laboratory-Based Enteric Disease Surveillance data.

This work was supported by the Centers for Disease Control and Prevention and the US Food and Drug Administration Center for Veterinary Medicine.

\section{About the Author}

Dr. Medalla is an epidemiologist with the National Center for Emerging and Zoonotic Infectious Diseases, Division of Foodborne, Waterborne, and Environmental Diseases, Centers for Disease Control and Prevention. Her research interests include antimicrobial resistance in Salmonella and other foodborne and enteric pathogens. 


\section{References}

1. Scallan E, Hoekstra RM, Angulo FJ, Tauxe RV, Widdowson MA, Roy SL, et al. Foodborne illness acquired in the United States - major pathogens. Emerg Infect Dis. 2011;17:7-15. https://doi.org/10.3201/eid1701.P11101

2. Pegues DA, Miller SI. Salmonella Species. In: John E. Bennett, Raphael Dolin, Blaser. MJ, eds. Mandell, Douglas, and Bennett's principles and practice of infectious diseases. Philadelphia: Elsevier Saunders; 2020. p. 2725-36.

3. Varma JK, Greene KD, Ovitt J, Barrett TJ, Medalla F, Angulo FJ. Hospitalization and antimicrobial resistance in Salmonella outbreaks, 1984-2002. Emerg Infect Dis. 2005;11:943-6. https://doi.org/10.3201/eid1106.041231

4. Krueger AL, Greene SA, Barzilay EJ, Henao O, Vugia D, Hanna S, et al. Clinical outcomes of nalidixic acid, ceftriaxone, and multidrug-resistant nontyphoidal Salmonella infections compared with pansusceptible infections in FoodNet sites, 2006-2008. Foodborne Pathog Dis. 2014;11:335-41. https:/ / doi.org/10.1089/fpd.2013.1642

5. Varma JK, Molbak K, Barrett TJ, Beebe JL, Jones TF, Rabatsky-Ehr T, et al. Antimicrobial-resistant nontyphoidal Salmonella is associated with excess bloodstream infections and hospitalizations. J Infect Dis. 2005;191:554-61. https://doi.org/10.1086/427263

6. Crump JA, Barrett TJ, Nelson JT, Angulo FJ. Reevaluating fluoroquinolone breakpoints for Salmonella enterica serotype Typhi and for non-Typhi salmonellae. Clin Infect Dis. 2003;37:75-81. https://doi.org/10.1086/375602

7. Clinical and Laboratory Standards Institute. Performance standards for antimicrobial susceptibility testing, 31st edition (M100). Wayne (PA): The Institute; 2021.

8. Johnson LR, Gould LH, Dunn JR, Berkelman R, Mahon BE; FoodNet Travel Working Group. Salmonella infections associated with international travel: a Foodborne Diseases Active Surveillance Network (FoodNet) study. Foodborne Pathog Dis. 2011;8:1031-7. https://doi.org/10.1089/ fpd.2011.0854

9. Dewey-Mattia D, Manikonda K, Hall AJ, Wise ME, Crowe SJ; Centers for Disease Control and Prevention. Surveillance for foodborne disease outbreaks - United States, 2009-2015. MMWR Surveill Summ. 2018;67:1-11. https://doi.org/10.15585/mmwr.ss6710a1

10. Kozlica J, Claudet AL, Solomon D, Dunn JR, Carpenter LR. Waterborne outbreak of Salmonella I 4,[5],12:i:-. Foodborne Pathog Dis. 2010;7:1431-3. https://doi.org/ 10.1089/fpd.2010.0556

11. Marder Mph EP, Griffin PM, Cieslak PR, Dunn J, Hurd S, Jervis R, et al.; Centers for Disease Control and Prevention. Preliminary incidence and trends of infections with pathogens transmitted commonly through food Foodborne Diseases Active Surveillance Network, 10 U.S. Sites, 2006-2017. MMWR Morb Mortal Wkly Rep. 2018;67:324-8. https://doi.org/10.15585/mmwr.mm6711a3

12. Centers for Disease Control and Prevention. Multistate outbreaks of Salmonella infections linked to contact with live poultry in backyard flocks, 2018 (final update). 2018 Sep 13 [cited 2020 Oct 29]. https://www.cdc.gov/salmonella/ backyard-flocks-06-18/index.html

13. Huang JY, Patrick ME, Manners J, Sapkota AR, Scherzinger KJ, Tobin-D'Angelo M, et al. Association between wetland presence and incidence of Salmonella enterica serotype Javiana infections in selected US sites, 2005-2011. Epidemiol Infect. 2017;145:2991-7. https://doi.org/10.1017/ S0950268817001790

14. Dechet AM, Scallan E, Gensheimer K, Hoekstra R, Gunderman-King J, Lockett J, et al.; Multistate Working
Group. Outbreak of multidrug-resistant Salmonella enterica serotype Typhimurium definitive type 104 infection linked to commercial ground beef, northeastern United States, 2003-2004. Clin Infect Dis. 2006;42:747-52. https://doi.org/ $10.1086 / 500320$

15. Varma JK, Marcus R, Stenzel SA, Hanna SS, Gettner S, Anderson BJ, et al. Highly resistant Salmonella Newport-MDRAmpC transmitted through the domestic US food supply: a FoodNet case-control study of sporadic Salmonella Newport infections, 2002-2003. J Infect Dis. 2006;194:222-30. https:// doi.org/10.1086/505084

16. Centers for Disease Control and Prevention. National Antimicrobial Resistance Monitoring System for Enteric Bacteria (NARMS): human isolates final report, 2015. Atlanta: The Centers; 2018.

17. Centers for Disease Control and Prevention. National Salmonella Surveillance [cited 2020 Oct 29]. https:/ / www. cdc.gov/nationalsurveillance/salmonella-surveillance.html

18. Centers for Disease Control and Prevention. National Antimicrobial Resistance Monitoring System for Enteric Bacteria [cited 2020 Oct 29]. https:/ / www.cdc.gov/narms/ reports/index.html

19. Medalla F, Hoekstra RM, Whichard JM, Barzilay EJ, Chiller TM, Joyce K, et al. Increase in resistance to ceftriaxone and nonsusceptibility to ciprofloxacin and decrease in multidrug resistance among Salmonella strains, United States, 1996-2009. Foodborne Pathog Dis. 2013;10:302-9. https://doi.org/10.1089/fpd.2012.1336

20. Medalla F, Gu W, Mahon BE, Judd M, Folster J, Griffin PM, et al. Estimated incidence of antimicrobial drug-resistant nontyphoidal Salmonella infections, United States, 2004-2012. Emerg Infect Dis. 2016;23:29-37. https://doi.org/10.3201/ eid2301.160771

21. Greene SK, Stuart AM, Medalla FM, Whichard JM, Hoekstra RM, Chiller TM. Distribution of multidrug-resistant human isolates of MDR-ACSSuT Salmonella Typhimurium and MDR-AmpC Salmonella Newport in the United States, 2003-2005. Foodborne Pathog Dis. 2008;5:669-80. https:/ / doi.org/10.1089/fpd.2008.0111

22. Crim SM, Chai SJ, Karp BE, Judd MC, Reynolds J, Swanson KC, et al. Salmonella enterica serotype Newport infections in the United States, 2004-2013: increased incidence investigated through four surveillance systems. Foodborne Pathog Dis. 2018;15:612-20. https://doi.org/10.1089/fpd.2018.2450

23. Jones TF, Ingram LA, Cieslak PR, Vugia DJ, Tobin-D'Angelo M, Hurd S, et al. Salmonellosis outcomes differ substantially by serotype. J Infect Dis. 2008;198:109-14. https://doi.org/ $10.1086 / 588823$

24. US Food and Drug Administration. 2018 NARMS update: integrated report summary interactive version [cited 2020 Dec 28]. https:/ / www.fda.gov/animal-veterinary/nationalantimicrobial-resistance-monitoring-system/2018-narmsupdate-integrated-report-summary-interactive-version

25. US Census Bureau. Population and housing unit estimates [cited 2020 Oct 29]. http:/ / www.census.gov/popest

26. Gu W, Medalla F, Hoekstra RM. Bayesian hierarchical model of ceftriaxone resistance proportions among Salmonella serotype Heidelberg infections. Spat Spatio-Temporal Epidemiol. 2018;24:19-26. https://doi.org/10.1016/ j.sste. 2017.10 .003

27. Lunn DJ, Thomas A, Best N, Spiegelhalter D. WinBUGSa Bayesian modelling framework: concepts, structure, and extensibility. Stat Comput. 2000;10:325-37. https:/ / doi.org/10.1023/A:1008929526011

28. Lambert PC, Sutton AJ, Burton PR, Abrams KR, Jones DR. 
How vague is vague? A simulation study of the impact of the use of vague prior distributions in MCMC using WinBUGS. Stat Med. 2005;24:2401-28. https:/ / doi.org/ 10.1002/sim. 2112

29. Chai SJ, White PL, Lathrop SL, Solghan SM, Medus C, McGlinchey BM, et al. Salmonella enterica serotype Enteritidis: increasing incidence of domestically acquired infections. Clin Infect Dis. 2012;54(Suppl 5):S488-97. https://doi.org/10.1093/cid/cis231

30. Marcus R, Varma JK, Medus C, Boothe EJ, Anderson BJ, Crume T, et al.; Emerging Infections Program FoodNet Working Group. Re-assessment of risk factors for sporadic Salmonella serotype Enteritidis infections: a case-control study in five FoodNet Sites, 2002-2003. Epidemiol Infect. 2007;135:84-92. https:/ / doi.org/10.1017/S0950268806006558

31. O'Donnell AT, Vieira AR, Huang JY, Whichard J, Cole D, Karp BE. Quinolone-resistant Salmonella enterica serotype Enteritidis infections associated with international travel. Clin Infect Dis. 2014;59:e139-41. https:/ / doi.org/10.1093/ cid/ciu505

32. Grass JE, Kim S, Huang JY, Morrison SM, McCullough AE, Bennett C, et al. Quinolone nonsusceptibility among enteric pathogens isolated from international travelers Foodborne Diseases Active Surveillance Network (FoodNet) and National Antimicrobial Monitoring System (NARMS), 10 United States sites, 2004-2014. PLoS One. 2019;14:e0225800. https://doi.org/10.1371/journal.pone.0225800

33. Harvey RR, Friedman CR, Crim SM, Judd M, Barrett KA, Tolar B, et al. Epidemiology of Salmonella enterica serotype Dublin infections among humans, United States, 1968-2013. Emerg Infect Dis. 2017;23:1493-501. https://doi.org/10.3201/eid2309.170136

34. Elnekave E, Hong S, Mather AE, Boxrud D, Taylor AJ, Lappi V, et al. Salmonella enterica serotype 4,[5],12:i:- in swine in the United States Midwest: an emerging multidrug-resistant clade. Clin Infect Dis. 2018;66:877-85. https://doi.org/10.1093/cid/cix909

35. Rabatsky-Ehr T, Whichard J, Rossiter S, Holland B, Stamey K, Headrick ML, et al.; NARMS Working Group. Multidrug-resistant strains of Salmonella enterica Typhimurium, United States, 1997-1998. Emerg Infect Dis. 2004;10:795-801. https://doi.org/10.3201/ eid1005.030209

36. Leekitcharoenphon P, Hendriksen RS, Le Hello S, Weill FX, Baggesen DL, Jun SR, et al. Global genomic epidemiology of Salmonella enterica Serovar Typhimurium DT104. Appl Environ Microbiol. 2016;82:2516-26. https:/ / doi.org/ 10.1128/ AEM.03821-15

37. Centers for Disease Control and Prevention. Multistate outbreak of multidrug-resistant Salmonella I 4,[5],12:i:- and Salmonella Infantis infections linked to pork (final update) [cited 2020 Oct 29]._https:/ / www.cdc.gov/salmonella/ pork-08-15/index.html

38. US Department of Agriculture, National Agricultural Statistics Service. Quarterly hogs and pigs. 2021 Mar 25 [cited 2021 Apr 19]. https:/ / downloads.usda.library.cornell. edu/usda-esmis/files/rj430453j/7p88db205/mw22w1890/ hgpg0321.pdf

39. US Department of Agriculture, Economic Research Service. Factors affecting U.S. pork consumption/LDP-M-130-01. 2005 May [cited 2020 Oct 29]. https:/ / www.ers.usda. gov/webdocs/outlooks/37377/15778_ldpm13001_1_. pdf? $v=5280.8$
40. US Department of Commerce, National Travel and Tourism Office. U.S. travel and tourism statistics (U.S. resident outbound) [cited 2020 Oct 29]. https:/ / travel.trade.gov/outreachpages/outbound.general_ information.outbound_overview.asp

41. Threlfall EJ, Day M, de Pinna E, Charlett A, Goodyear KL. Assessment of factors contributing to changes in the incidence of antimicrobial drug resistance in Salmonella enterica serotypes Enteritidis and Typhimurium from humans in England and Wales in 2000, 2002 and 2004. Int J Antimicrob Agents. 2006;28:389-95. https:/ / doi.org/ 10.1016/j.ijantimicag.2006.07.009

42. Bae D, Kweon $\mathrm{O}$, Khan AA. Isolation and characterization of antimicrobial-resistant nontyphoidal Salmonella enterica serovars from imported food products. J Food Prot. 2016;79:1348-54. https:/ / doi.org/10.4315/0362-028X.JFP-15-564

43. Karp BE, Campbell D, Chen JC, Folster JP, Friedman CR. Plasmid-mediated quinolone resistance in human nontyphoidal Salmonella infections: an emerging public health problem in the United States. Zoonoses Public Health. 2018;65:838-49. https://doi.org/10.1111/zph.12507

44. Centers for Disease Control and Prevention. Foodborne Diseases Active Surveillance Network (FoodNet): FoodNet 2015 surveillance report (final update). Atlanta: The Centers; 2017.

45. Scallan E, Crim SM, Runkle A, Henao OL, Mahon BE, Hoekstra RM, et al. Bacterial enteric infections among older adults in the United States: Foodborne Diseases Active Surveillance Network, 1996-2012. Foodborne Pathog Dis. 2015;12:492-9. https:// doi.org/10.1089/fpd.2014.1915

46. Angelo KM, Reynolds J, Karp BE, Hoekstra RM, Scheel CM, Friedman C. Antimicrobial resistance among nontyphoidal Salmonella isolated from blood in the United States, 20032013. J Infect Dis. 2016;214:1565-70. https:/ / doi.org/ 10.1093/infdis/jiw415

47. Crump JA, Medalla FM, Joyce KW, Krueger AL, Hoekstra RM, Whichard JM, et al.; Emerging Infections Program NARMS Working Group. Antimicrobial resistance among invasive nontyphoidal Salmonella enterica isolates in the United States: National Antimicrobial Resistance Monitoring System, 1996 to 2007. Antimicrob Agents Chemother. 2011;55:1148-54. https:/ / doi.org/10.1128/AAC.01333-10

48. Boore AL, Hoekstra RM, Iwamoto M, Fields PI, Bishop RD, Swerdlow DL. Salmonella enterica infections in the United States and assessment of coefficients of variation: a novel approach to identify epidemiologic characteristics of individual serotypes, 1996-2011. PLoS One. 2015;10:e0145416. https:// doi.org/10.1371/journal.pone.0145416

49. McDermott PF, Tyson GH, Kabera C, Chen Y, Li C, Folster JP, et al. Whole-genome sequencing for detecting antimicrobial resistance in nontyphoidal Salmonella. Antimicrob Agents Chemother. 2016;60:5515-20. https://doi.org/10.1128/ AAC.01030-16

50. Karp BE, Tate H, Plumblee JR, Dessai U, Whichard JM, Thacker EL, et al. National Antimicrobial Resistance Monitoring System: two decades of advancing public health through integrated surveillance of antimicrobial resistance. Foodborne Pathog Dis. 2017;14:545-57. https:// doi.org/10.1089/fpd.2017.2283

Address for correspondence: Felicita Medalla, Centers for Disease Control and Prevention, 1600 Clifton Rd NE, Mailstop H24-9, Atlanta, GA 30329-4027, USA; email: fmedalla@cdc.gov 